\title{
Sicherheit durch Automatisierung
}

\section{Liebe Leserin, lieber Leser,}

ebenso wichtig wie Zero Emissions ist die Vision Zero - das Ziel eines Straßenverkehrs ohne Opfer. Die Europäische Union will, dass 2050 nahezu niemand mehr auf europäischen Straßen stirbt. Hierzu soll die Zahl der Getöteten im Verkehr innerhalb eines jeden Jahrzehnts halbiert werden. Ein ambitioniertes Ziel, denn 2016 starben EU-weit mit 25.500 Personen im Straßenverkehr nur 600 weniger als im Vorjahr - ein Rückgang von erst $19 \%$ seit 2010 .

Bei der passiven Sicherheit von Fahrzeugen wurde viel erreicht. Wichtig ist nun, prädiktiv auf das Unfallgeschehen einzuwirken. Die Technologiesprünge bei der Entwicklung vom teil- zum hochautomatisierten Fahren ermöglichen kooperative Sicherheitstechniken in Fahrzeugen und der Verkehrsinfrastruktur. Sie erlauben automatisierte Reaktionen, die besonders in Kreuzungsbereichen oder bei plötzlich auftretenden Gefahrensituationen Unfälle verhindern oder zumindest deren Folgen abmildern. Allerdings besteht kein Grund zur Euphorie, denn noch wird an der Einführung von Level 3 gearbeitet - bis zu Level 4 oder Level 5 ist es noch ein weiter Weg. Die Technologien werden zuerst wohl in Oberklassefahrzeugen eingeführt werden, sodass es noch lange dauern wird, bis sie in die gesamte Fahrzeugflotte kaskadiert sein werden. Die Berliner Erklärung zur Verkehrssicherheit des VDI zeigt aber, dass Experten intensiv auf das Thema fokussieren und die Betrachtung von Einzelmerkmalen zur Sicherheit immer mehr einer Betrachtung von Fahrzeug und Umfeld als Gesamtsystem weicht.
Bertrandt bilanziert in dieser ATZ, was der Weg zum vollautomatisierten Fahren für die Entwicklung der Rückhaltesysteme bedeuten kann. Scania stellt einen neuartigen Vorhangairbag für schwere Nutzfahrzeuge vor. Prof. Rodolfo Schöneburg diskutiert im Interview die Trends in der Entwicklung von Sicherheitssystemen und die Notwendigkeit von Crashtests. Ob Gurt, Airbag oder Sensoren - neue Einsatzszenarien bewirken eine andere Bauteilauslegung. Der Weg zum automatisierten Fahren bringt Bewegung und ein anderes Denken in die Sicherheitstechnik. Automatisierung erhöht die Sicherheit im Straßenverkehr, wenn nicht das Fahrzeug im Vordergrund steht, sondern Mobilität als Gesamtsystem gedacht und vor allem zügig gehandelt wird.

Ich wünsche Ihnen viel Spaß bei der Lektüre der aktuellen ATZ.

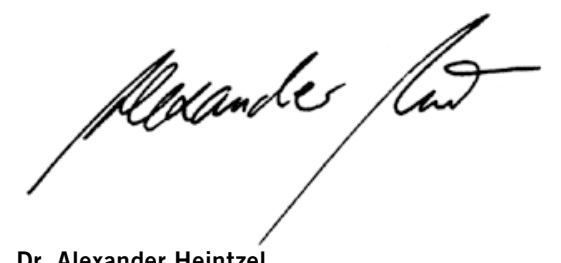

Alexander Heintzel

Chefredakteur

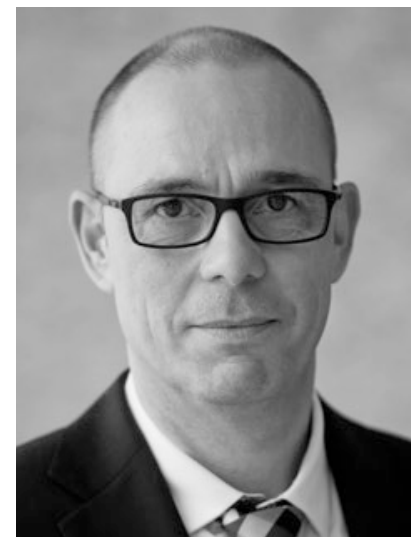

\section{Ihr Partner für den virtuellen Fahrversuch}

\section{Als Technologietreiber die automobile Zukunft mitgestalten}

Mit unseren Lösungen helfen wir als Technologieführer für den virtuellen Fahrversuch unseren Kunden weltweit realitätsgetreue virtuelle Tests im gesamten Entwicklungsprozess gemäß dem Prinzip des Automotive Systems Engineering durchzuführen und somit reale Prototypen einzusparen. 